\title{
A Survey of Computational Toxicology Approaches
}

\author{
Dr. Amena Mahmoud \\ Assistant Professor at Department of Computer Sciences, faculty of Computers and Information, Kafr \\ El Sheikh University, Egypt. \\ E.mail: $\underline{\text { amena_mahmoud@fci.kfs.edu.eg }}$
}

\begin{abstract}
Medications are a particular kind of chemicals that are considered essential for toxicity screening in contrast to those substances that contribute to the environment. In the development and production phases, toxicity is still the reason for a great number of candidate failures for new medicines. In-vivo work on the pharmaceutical industry, the in-vitro and animal trends of incompetence for correctly forecasting certain human toxicity, and the lack of accurate, high-thrown in vitro testing was obstructive in calculating toxicity. the development of computational toxicology structures has been encouraged by developing numerous "omics" techniques that have grown into several scientific areas, including genomics, proteomics, metabolomics, and transcriptomics. Computational toxicology is highly interdisciplinary. Researchers in the field have backgrounds and training in toxicology, biochemistry, chemistry, environmental sciences, mathematics, statistics, medicine, engineering, biology, computer science, and many other disciplines. This paper offers a historical perspective and current status for the computational approaches used at the assessment of toxicity. It presents examples of the expert systems, machine-learning approaches and web-based toxicity predictors.
\end{abstract}

Keywords - Computational Toxicology, In Silico Modeling, Expert Systems, Machine-Learning Approaches.

\section{Introduction}

There are over 80,000 chemicals in common use worldwide, and hundreds of new chemicals and chemical mixtures are introduced into commerce each year. Because chemical safety has traditionally been assessed using expensive and time-consuming animalbased toxicity tests, only a small fraction of these chemicals has been adequately assessed for potential risk.

Potential methodologies to conventional toxicity testing are critical for environmental and pharmaceutical products, so that toxicity potential can be estimated and research priority is given the limited resources available. Another such method is robot toxicology.

Computational Toxicology is described by the U.S. Environmental Protection Agency (EPA) as " the use of Software and mathematical models to forecast negative impacts and to better understand the person or several processes through which a chemical cause harmful effect."

In a broader context, computational toxicology is an emerging multidisciplinary field that combines knowledge of toxicity mechanisms with appropriate biological and chemical information to allow the design, development, and testing of multi-scale machinebased learning methods that are used to gain knowledge in the pathways through which a chemical causes damage. In-vivo toxicology also seeks to use highinformation content data, advanced biostatistical approaches, and computational complexity to analyze these data to handle and identify patterns and associations in large biological and chemical data sets.

Some of the main areas of application of computational toxicology are hazard and danger prioritization of chemicals, discovery of useful mechanical knowledge in tailoring testing programs for each chemical, health screening of food additives and food contact substances, aid of more advanced aggregate and cumulative risk assessment methods, calculation of risk assessment, pharmaceutical lead choice in drug development, pharmaceutical contaminant safety testing and certification, and drug metabolite safety screening.

Computational toxicology is highly interdisciplinary. Researchers in the field have backgrounds and training in toxicology, biochemistry, chemistry, environmental sciences, mathematics, statistics, medicine, engineering, biology, computer science, and many other disciplines. 


\section{Computational Toxicology}

In order to identify the relevance of both chemical composition and toxicological end punctures, computer toxicology is a comparatively novel area of applicable science that integrates fundamental principles from biology, chemistry, and informatics. In addition, these interactions can be used to estimate the toxicity of unproven substances. In the middle of the 19th and early 20th centuries, crucial scientific breakthroughs were explained by the creation of a physical foundation for molecular structure and the association of chemicals with biological systems.

As early as 1858 Borodin, a Russian chemist and composer [1], while referring to compounds and their properties quoted, "their toxicological properties and chemical makeup are closely related." However, it is the codification of the then-known elements into the Periodic Table in the late 1880s by Julius Lothar Meyer and Dmitriy Mendeleev [2] that can rightly be regarded as the beginning of similarity-based clustering of chemical elements. This grouping formed the basis for predictive chemistry and found use not only for predicting the reactive properties of the elements but also laid the groundwork for understanding, and ultimately for being able to predict, more complex physicochemical properties of combinations of elements.

This work quickly followed the discovery of statistical relationships amongst toxicity and basic physiochemically belongings for minor groups of particles. In the year 1863 Cros, for instance, discovered that the paralysis characteristics of a series of cauterized strychnine are based on the nature of a quaternation Group [3], Cros found, for example, an inverse relationship between mammaliany liquor poisonousness and water problems [4]. In his research [5] he noted a correlation between the poisonousness of aquatic solubility and unassuming biological complexes, and advanced they found independently in their research [6] that the tranquillizer activity of numerous mixtures remained grounded on their oil-water panel constant.

The development by Gilbrt Newton Leweis [7] at the turn of the 20th century of the conduction principle of chemical bond formation and of transmitter hypothesis based on Langley's ground breaking work [8] established physical association theoretical constructs amongst xenobiotics and biological macromolecules. Valence hypothesis developed the idea that selective physicochemical ligand transmitter interactions can only lead to a specific genetic reaction with specific toxicants or drug compounds (or with functional groups of these compounds).
A great deal of experimental work quickly revealed the majority of the pharmaceutical products and toxicants in a receptor-mediate manner for exploit and offered a balanced source intended for the connection between structure and function. Hammet provided a linear and free energy equation established in the 1930s [9] with his groundbreaking exertion arranged the quantifications of consequence of substituent on estery hydrolysisal as the thermodynamic basis for parametric operations that explain the interaction of the structure with the behavior, this includes an analysis of unique possessions fashionable rapports of molecular structure descriptive parameters. Taft's use of steric and hydrophilicity identifiers decadently confirms not only the significance of free linear energy links [10], but also the theory of quantitative structure-activeness relationships "effect", the term "effect" for any physicochemical, pharmacological or toxicological components theory of quantity-activity relationships.

During the process, digital computers were becoming more available and efficient. Scholarly innovators [11] using the principles of linear free energy related approaches to use the data of toxicity that are then accessible, started exploring the responsibilities of various methods and identifiers of chemical composition. The birth of the science of computational toxicology was marked by this convergence of statistical analysis, chemical toxicology, and computer technology.

Continued advances in sophisticated computational and statistical algorithms today give toxicologists access to a host of new approaches and tools to help them perform and manage structure activity relationship analyses for toxicologically important endpoints. The principal differences among the various computational methods arise from the way a molecule is quantified in terms of descriptors and how the relationship between these chemical descriptors and the toxicological endpoint of interest is established. For example, chemical structure descriptors range from measured or computed physical properties, such as $\operatorname{logP}$ (the logarithm of the n-octanol-water partition coefficient), number of Hbond donors, and molecular weight, to all possible continuous pieces or "fragments" of molecules, to descriptors that represent the electronic configurations or surface maps of the whole or localized portions of the molecule.

Similarly, an in-silico toxicology solution can be a generic expert model or a machine learning system with an algorithm that tests empirically the presence of a statistically significant correlation between an important toxicological outcome and one or more descriptors. Brief historical records are given in the following sections for each of these major strategies. 


\subsection{Methods of In Silico Modeling}

Various chemical toxicity calculation software approaches have been developed. Because either they support the historical origins of toxicology at a lifespan or because they reflect the actual toxicity prediction mechanism, the tools we discuss here are chosen. Possible strategies for using the approach, statistical description, strength and weakness evaluation, guidelines on how and when the system is to be used, and are provided in each solution (if applicable). Therefore, for consistency purposes, we retain as common formulas and graphical interpretations of structures as appropriate.

\section{Rule-based Models and Structural Alerts}

Structural alerts [12] are chemical structures that demonstrate or are associated with toxicity. Structural alerts may take the form only of a single atom or several linked atoms. A confluence of structural alerts may make a contribution more than a single structural alert to toxicity. Structural alerts are being used often in rules determined in the form ' if $\mathrm{A}$ is $\mathrm{B}$ then $\mathrm{T}$, ' which $\mathrm{A}$ is a structural alert, B is the valuation of the structural alert, and $\mathrm{T}$ is the toxicity prediction with the assigned level of certainty.

Structural alerts are easily interpreted and implemented [13]. They are useful in the design of drugs to determine how drugs should be altered in order to decrease their toxicity. The use of the Toxicity Predicting Model enables the detection of the model of possible metabolites. Furthermore, there are a number of limitations to structural alerts. Structural alerts only use quantitative characteristics (e.g. chemical components are either available or missing) or subjective endpoints (e.g. carcinogenic and noncarcinogenic). Structural alerts don't provide perspective into the toxicity of biological pathways and may not be enough to predict toxicity. Toxicity may decrease or increase depending on the absence or presence of other chemical properties at the same time. The set of structural alerts and guidelines may be insufficient, leading to a significant number of false negatives in predictions (i.e. toxic chemicals expected as non-toxic).

The last section is especially important. How to view the performance of structural alerts frameworks should be understood. This does not mean non-toxicity if a chemical does not include structural warnings or does not comply with any toxicity laws [14]. That's particularly the case for human-based rules that typically involve structural alerts or rules indicating toxicity but do not include structural alerts or rules indicating non-toxicity. Therefore, when designing these models, it is necessary to ensure that the list of structural alerts and rules are extensive and updated as more observational data

become accessible. Moreover, the list of structural alerts and rules, their comprehensiveness and predictive power should be balanced. When structural alerts and guidelines are varying, they can be extended to a vast amount of chemicals, but this may raise false positives (i.e., expected as dangerous non-toxic chemicals).

Read-Across, Trend Analysis, and Chemical Category

A chemical category [15] is a class of chemicals with different or identical properties and toxicity effects. The OECD Guidance on Classification of Chemicals lists many classification approaches, including chemical identification and structure, physicochemical and ADME properties, chemical / biological interactions and action mechanism. The OECD recommendations define structural similarities as the point of reference for grouping, but it is also disputed for missing a ' scientifically provable rationale ' for grouping and may be used when particulates or other components in the chemical structure do not affect toxicity.

Read-across is a technique for predicting a chemical's unknown toxicity using similar chemicals (known as chemical analogs) from the same chemical category [15]. Statistical analysis is a method of predicting a chemical's toxicity by analyzing trends in toxicity (increasing, decreasing, or constant) of the chemicals tested. A specific example of statistical analysis showed an increase in acute aquatic exposure as the length of the carbon chain increases. Fig.1 gives a description of the various parameters to be addressed when constructing a read-across system.

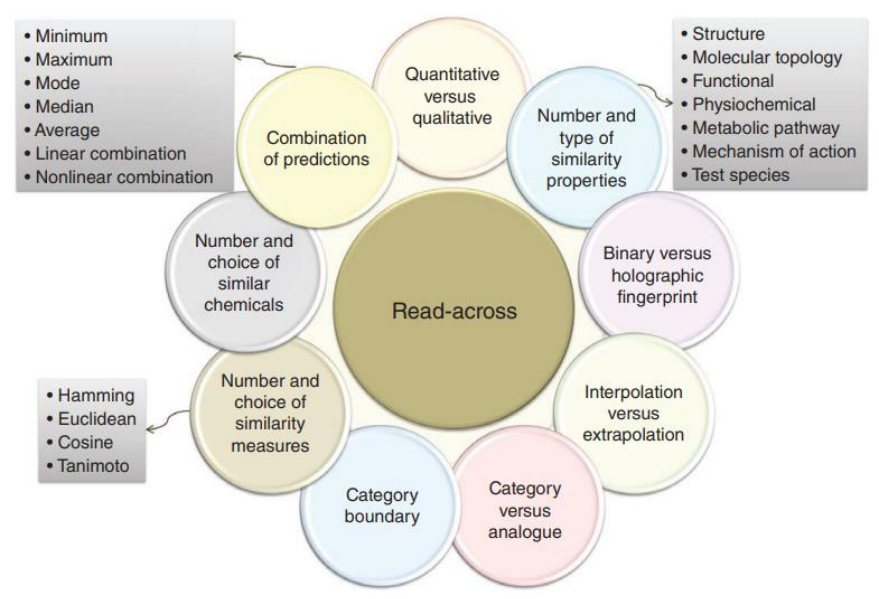

Fig.1. Different properties of read-across models [16]

There have been two methods to develop a readacross approach [17]: analog approach (known as onetone), using one or a few analogs, and a class approach (known as many-to-one) which incorporates multiple 
analogs. Since two analogs may have different toxicity profiles, the analog methodology may be prone to

outliers. Using multiple category approach analogs are helpful in identifying patterns within a category and may increase confidence in predictions of toxicity. Classification strategy demands the creation of a boundary classification to decide whether a chemical belongs to the class and the introduction of a system for ' combining estimates ' of analogs with different toxicity profiling.

\section{TOPKAT}

The very commonly utilized approaches of evaluating a chemical's possible carcinogenicity are: chronic bioassays for carcinogenicity including rodents; genotoxicity studies in mammalian cells, microbes, or intact mammals; and applying the theories of structureactivity relationships to forecast carcinogenicity on the basis of observed chemical testing results with a similar concept. Human experts [18] or computers can carry out structure activity relationship analysis.

Computer-based approaches fall into two basic categories: those which seek to understand human expert reasoning processes [19] and others that conduct statistical equivalences in the database of the system between the characteristics of the chemical of interest and those of known carcinogens and noncarcinogens [20]. One of these latter types of widely used systems is TOPKAT (Toxicity Prediction by Komputer Assisted Technology). The utility of the TOPKAT carcinogenicity unit was measured by evaluating the ability of the program to predict the effects of the National Toxicology Program's rodent carcinogenicity bioassays.

Following a successful method with a number for endpoints of toxicity in 1987, HDi introduced TOPKAT, Komputer Assisted Technology's first QSAR-based toxicity predictor application currently being sold by Accelrys, Inc. This latest version, TOPKAT 6.2, provides high detail electromagnetics, geometry, and configuration identifiers rather than a pre-defined substructures kit, that require the measurement of 16 specific toxicity endpoints for prepackaged comprehensive QSARs and the learning databases that have been extracted from QSARs.

\subsection{Expert Systems}

Simply stated, expert systems are computer applications that carry out a degree of logical reasoning similar to those of human beings, making them ideal systems for making subject-matter expertise available to non-experts Relying on mathematical and logic techniques, an expert system is comprised of two main components: (1) a Knowledge Base (KB); and (2) an Inference Engine (IE) [21].

Later maintains the knowledge of the subject in the form of a number of regulations - formalized facts derived from true experience - and later, by logically combining the rules caused by feedback, makes acceptable deductions. Active, and widely used in many fields, expert systems are artificial intelligence systems, including clinical diagnostics, credit permits, genetic engineering, airline scheduling, analytical chemistry, chemical structures, computer toxicology etc. [22].

As a computer-discernible set of often mostly nested IF-THEN structures, construction poisonousness relations known besides reliable by skilled toxicologists are contained in a digital toxicology expert network. The expert model analyzes its composition when asked to determine the toxicity of a substance and makes correlations with the existing KB of rules. For analytical deductive evaluation of possible toxicity, the laws caused by the composition of the compound are gathered and introduced to the IE.

The need for expert systems in the diverse field of toxicology in the pharmaceutical industry is long overdue as the toxicology expertise is not sufficient to satisfy the demand - definitely not, where and when appropriate, in regulatory authorities or in drug research teams. In the scope of the study of compound toxicity rates, a framework for statistical toxicology specialists in these organizations will provide a range of toxicological expertise.

The competence and knowledge of the research community provides the $\mathrm{KB}$ with the guidelines in a statistical toxicology framework. As stated, even though the mid-1800s methodological associations are recognized among toxicological and chemical composition. Further systematic comparisons between physicochemical and toxicological properties provide validated $\mathrm{KB}$ learning inputs to an expert framework along with the proven carcinogenicity consequences of congeneric substances in small ligands, such as benz [c] acridine methyl variants, which are typical of greater carcinogenicity than benz acridine methyl derivatives [23].

For more medical and epidemiologic evidence on toxicity, toxicologists are looking for clear interrelationships between toxic effects and molecular structures and/or properties. There are three major toxicology specialist programs available on the market, namely HazardExpert [24], Oncologic [25], and DEREK [26]. 


\section{HazardExpert}

HazardExpert is CompuDrug Chemistry Ltd.'s first computer-based toxicity forecasting expert system [27] established in 1985. HazardExpert predicts a variety of toxicity endpoints from carcinogenic, irritable, immunetoxic, neurotoxic, teratogenic, and mutagenic. HazardExpert's KB includes Toxic Fragments Knowledge Base from the literature on structuretoxicity interactions and documents from the U.S. EPA reports. Based on the impact of these fragments on biological systems, the predictive principles in HazardExpert combine expert intuition and fuzzy logic.

\section{OncoLogic}

LogiChem, Inc. created this consulting program under a cooperation agreement between the Pollution Prevention and Toxics Office of the U.S. EPA [28]. Carcinogencity is OncoLogic's primary toxicity target being predicted. Its instruction corrupt is structural alerts whereas the carcinogenicity technique is well understood in humans and animals. The ability of OncoLogic to predict the carcinogencity of both organic compounds and polymers, metals and fibers represents an important benefit. From the USA OncoLogic rights have been covered by EPA, it can be commonly used in the future.

\section{DEREK}

Schering Agrochemical Corporation, the premier founder of the expert system DEREK, donated the system to its regular non-profit provider, Lhasaa Imperfect [29]. Considering a compound's chemical structure, DEREK expects a range of toxicological risks, including genotoxicity, carcinogenicity, skin resistance and discomfort, based on the respective knowledge base built from the shared experience of scientists from more than 20 member organizations.

The KB is usually driven by various combinations of structurally toxic guidelines or systemic ' alerts ' due to differences in compound count as well as in the interpretation and the comprehension of analytical results. There are, for example, 96 guidelines on genotoxicity evaluation yet only 47 for cancer prediction [30]. Rather than 70 mutagenic laws are also in place, but chromosome abomination is significantly lower. The laws are typically drawn from structures of organic chemistry identified by the toxic reaction found in a variety of associated substances.

DEREK is a rules-based model that evaluates the potential toxicity of a chemical structure in a subjective way only. One of the nine statistical classes are applied to each prediction: Sure, Probable, Plausible, Equivocal, Doubted, Improbable, Invalid, Possible, and Contradicted. Wherever appropriate, with specific literature links, the statement is further supported.

A number of independent data sets tested its performance.

\subsection{Machine-Learning Approaches}

So far, several in-silico approaches to the analysis of drug safety have been developed that can commonly be classified into three groups: quantitative correlation, qualitative grouping, and read-across. In assessing drug safety - as a beginning- we only demand to know that a product is, rather than its exact toxicity level, poisonous and highly toxic or moderately harmful. A measurable construction poisonousness association model may be developed for a small proportion of chemical analogs to estimate accurate toxicity values. Read-across is indeed an attainable technique to overcome these toxicity endpoints with experimental toxicity values from similar structures. Such systems have high accuracies, especially in local chemical spaces, and can sometimes substitute assays for similar endpoints in vitro or in-vivo. Structural alerts can actually be obtained from templates as a guide to a drug with adverse organs effects [31], which can be used in structural alteration to minimize the risk from chemists.

Random forest, Support vector machine, k-nearest neighbor, and boost tree are basic methods of machine learning commonly used in classification and regression models. Support vector machine, also known in particular as Support Vector Classifier or Support Vector Regression, is known for its high predictive reliability and lower overfitting risk [32]. The support vector machine 's basic idea is to build a hyperplane with the maximum distance to the nearest learning data points (support vectors) in a high-dimensional space. Random forest and boost tree come from the tree of choice [33].

Random forest could be seen as picking up most decision trees that use and merge a random subset of features through a voting system. Like Random forest, where each tree is equal, boost tree varies each tree's weight dynamically according to the mean prediction error. K-nearest neighbor is one of the simplest [34] algorithms. The principle of $\mathrm{k}$-nearest neighbor is that there are different biological properties in materials with similar structures. A result of the k-nearest neighbor is defined by the votes of its neighbors ' groups.

Very often the combination of these algorithms is applied to improve the performance of prediction approaches. A combined technique has been developed 
by means of a reproduction neural network procedure to accumulate the probability of final grouping conclusion, showing that the collective approaches are greater toward "single" methods [35].

Recently, in overcoming such problems as computer vision and speech recognition, deep learning has been applied [36]. One of the profound learning techniques is the multilayer neural network. Unlike the common artificial neural network, which has only three layers along with the input layer, hidden layer, and output layer, the multilayer neural network comprehends additional than individual concealed coating and is, therefore, more competent with complex mechanisms fashionable great toxicologically information. It can perform better than the artificial neural network and above-mentioned classical machine learning approaches when the training set is large [37].

Additional multifaceted network has extra weightiness for matching plus considerable supplementary undoubtedly to overfit. Recently, graphconvolutional networks [38] and enduring remembrance structures [39] were created to remove topographies since atomic particles and show better efficiency in managing thousands or more of compounds [40]. DeepChemi is an open-source, high-quality toolchain that makes it easier to use DL on the discovery of medicines and other areas.

\section{Conclusions}

Clearly, all computational toxicology approaches need to benefit from experimental data. Of our perspective, computational toxicology's most definite objective is not to remove the development and the use of standard, research lab-based biomarker strategies. On the contrary, the processes meant to be mutually complementary. Extension of the toxicological methods of experiments could be used to justify developing and assessing the safety of certain compounds, which may reduce the cost and production time of drug development in the pharmaceutical industry.

In addition, their prudent use by regulatory agencies to aid more rapid decision making, to be more effective in hazard assessment, and to assist in managing the environmental and human health effects of chemicals can be leveraged to great advantage in making sound rational choices regarding society's use of particular chemical entities, both now and in the future.

\section{References}

[1] Mendeleev D. "The periodic law of the chemical elements.", J Chem Soc. 1889; 55: pp. 634-56.

[2] Cros AFA. "Action de l'alcohol amylique sur l'organisme.", University of Strasbourg, 1863.

[3] Crum-Brown A, Fraser TR., "On the connection between chemical constitution and physiological action. Part 1. On the physiological action of the salts of the smmonium bases, derived from Strychnia, Brucia, Thebaia, Codeia, Morphia, and Nicotia.”, Trans R Soc Edinburgh, 1868-9; 25: pp. 151-203.

[4] Richardson BW., "Lectures on experimental and practical medicine. Physiological research on alcohols." Med Times Gaz, 1869; 2: pp. 703-6.

[5] Richet C., "Note sur le Rapport Entre la Toxicite et les Propriretes Physiques des Corps.”, Compt Rend Soc Biol, Paris, 1893; 45: pp. 775-6.

[6] Meyer H., "Zur theorie der alkoholnarkose.", Arch Exp Pathol Pharmakol, 1899; 42: pp. 109-18.

[7] Overton E., "Ueber die allgemeinen osmotischen Eigenschaften der Zelle, ihre vermutlichen Ursachen und ihre Bedeutung fur die Physiologie.", Vierteljahrsschr Naturforsch Ges Zurich, 1899; 44: pp. 88-114.

[8] Lewis GN., "The atom and the molecule.", J Am Chem Soc, 1916; 38: pp. 762-86.

[9] Langley JN., "On the contraction of muscle, chiefly in relation to the presence of 'receptor' substances.", Part IV. J Physiol, 1904; 33: pp. 374.

[10] Hammett LP., "Physical organic chemistry: Reaction rates, equilibria and mechanism", 2nd edition. New York: McGrawHill, 1970.

[11] Taft RW, Lewis IC., "Evaluation of resonance effects on reactivity by application of the linear inductive energy relationship: V. Concerning a sR scale of resonance effects". J Am Chem Soc, 1959; 81: pp. 5343.

[12] Hansch C, Maloney PP, Fujita T, Muir RM., "Correlation of biological activity of phenoxyacetic acids with Hammett substituent constants and partition coefficients.", Nature 1962; 194: pp. 178-80.

[13] Enslein K, Craig PN. “A toxicity prediction system.”, J Environ Toxicol, 1978; 2: pp.115-21.

[14] Smithing MP, Daravas F. "HazardExpert: An expert system for predicting chemical toxicity.", In: Finley, JW, Armstrong DJ, Robinson, SF, editors, Food safety assessment. Washington, DC: American Chemical Society, 1992, pp. 191-200.

[15] Hansch C, Maloney PP, Fujita T, Muir RM., "Correlation of biological activity of phenoxyacetic acids with Hammett substituent constants and partition coefficients.", Nature, 1962; 194: pp. 178-180.

[16] Enslein K, Lander TR, Tomb ME, Craig PN. "A predictive model for estimating rat oral LD50 values.”, In: Benchmark papers in toxicology, Vol 1. Princeton, NJ: Princeton Scientific Publishers, 1983.

[17] Enslein K, Blake BW, Tomb ME, Brgstedt HH. "Prediction of Ames test results by structure-activity relationships.", In vitro Toxicol, 1986;1: pp. 33-44.

[18] Luger GF. "Artificial intelligence: Structures and strategies for complex problem solving", 5th Edition. Boston: AddisonWesley, 2005.

[19] Kahn J. "From airports tarmacs to online job banks to medical labs, artificial intelligence is everywhere.", Wired, 2002;10 (3).

[20] Smith IA, Seybold PG. "Substituent effects in chemical carcinogens: Methyl derivatives of the benzacridines.", J Hetero Chem, 1979; 16: pp. 421-425.

[21] Woo Y, Lai D, Argus M, Arcos J. "Development of structureactivity relationship rules for predicting carcinogenic potential of chemicals.", Toxicol Lett, 1995; 79: pp. 219-228.

[22] Sanderson DM, Earnshaw CG. "Computer prediction of possible toxic action from chemical structure: The DEREK system.", Hum Exp Toxicol, 1991; 10: pp. 261-273.

[23] Sanderson DM, Earnshaw CG. "Computer prediction of possible toxic action from chemical structure: The DEREK system.", Hum Exp Toxicol, 1991; 10: pp. 261-273.

[24] Crettaz P, Benigni R. "Prediction of the rodent carcinogenicity 
of 60 pesticides by the DEREKfW expert system.", J Chem Inf Model, 2005; 45: pp. 1864-1873.

[25] Klopman G. "Artificial intelligence approach to structureactivity studies: Computer automated structure evaluation of biological activity of organic molecules.”, J Am Chem Soc, 1984; 106(24): pp. 7315-7321.

[26] Klopman G. "MULTICASE, A hierarchical computer automated structure evaluation program.", Quant Struct-Act Rel, 1992; 11(2): pp. 176-184.

[27] Japertas P, Didziapetris R, Petrauskas A. "Fragmental methods in the analysis of biological activities of diverse compound sets.”, Mini Rev Med Chem, 2003; 3(8): pp. 797-808.

[28] Zmuidinavicius D, Japertas P, Petrauskas A, Didziapetris R. "Progress in toxinformatics: The challenge of predicting acute toxicity." Curr Top Med Chem, 2003; 3(11): pp. 1301-1314.

[29] Kramer S, Helma, C. "Machine learning and data mining.", In: Helma C, editor, Predictive toxicology. Philadelphia: Taylor and Francis, 2005, pp. 223-254.

[30] De Castro L. and Zuben F. J., " Learning and Optimization Using the Clonal Selection Principle.”, IEEE Transactions on Evolutionary Computation, 2002, 3(6), pp. 239-251.

[31] Ji Z. and Dasgupta D., "Real-Valued Negative Selection Algorithm with Variable-Sized Detectors.”, LNCS 3102, Proceedings of GECCO, 2004, pp. 287-298.

[32] De Castro L. and Zuben F. J., " aiNET: An artificial immune network for data analysis.", Idea Group Publishing, Chapter XII, 2001, pp. 231-259.

[33] De Castro L. and Timmis J., "Artificial Immune Systems as a Novel Soft Computing Paradigm.", Soft Computing Journal, 2003,7(7).

[34] Dasgupta D. and Nino L., "Immunological Computation: Theory and Applications.", CRC Press, 2009.

[35] Hofmeyr S. A., "An Interpretative Introduction to the Immune System." Oxford University Press: New York, 2000.

[36 Parkin J. and Cohen B., "An Overview of the Immune System.”, The Lancet, 2001, 357.

[37] De Castro L. and Zuben F. J., "Artificial Immune Systems: Part I - Basic theory and applications." TR - DCA 01, 1999.

[38] Timmis J., Knight T., de Castro L. and Hart E., "An Overview of Artificial Immune Systems." , Natural Computation Series, 2004, pp. 51-86.

[39] Delahunty A. and Callaghan D., "Artificial Immune Systems for the Prediction of Corporate Failure and Classification of Corporate Bond Ratings.", Master of Management Science, University College Dublin, August, 2004.

[40] Timmis J., Hone A., Stibor T. and Clark E., "Theoretical Advances in Artificial Immune Systems.", Theoretical Computer Science, 2008, 1(403), pp.11-32. 